\title{
Hearing through a glass brightly
}

\section{Lawrence Challis and Stanley Clough}

To many, efforts to increase the efficiency with which sound can be amplified must seem a thoroughly antisocial activity. But this, of course, is audible sound where there is a well-tried route using a microphone to convert sound into electrical signals which are amplified and converted back to sound via loudspeakers. Ultrasound, at much higher frequencies, is extensively used in telecommunications, medical imaging and nondestructive testing; it is also a valuable probe for research on condensed matter, much of it carried out at low temperatures. Amplification at these frequencies of around $100 \mathrm{MHz}$ and above is again normally electronic, with conversion to and from ultrasonic waves via transducers. This may sound familiar: .it was the approach originally used in optical communication to amplify, every $100 \mathrm{~km}$ or so, the light intensity passing down a glass fibre. But then, in 1987, Gambling and colleagues at Southampton University showed that optical amplification could be achieved within a fibre by doping the glass and pumping it to achieve population inversion. Prieur, Höhler, Joffrin and Devaud ${ }^{1}$ have now shown that glass can also be made to amplify ultrasonic waves.

The first reports of ultrasonic amplification appeared in 1961. One technique, introduced by Tucker ${ }^{2}$, achieved amplification at $1.5 \mathrm{~K}$ by inverting the populations of two levels of a $\mathrm{Cr}^{3+}$ ion in $\mathrm{Al}_{2} \mathrm{O}_{3}$ (ruby) separated by the energy of the ultrasonic phonon, $\hbar \omega$. The ground state of this ion is split into a ladder of four separate levels ( 1 to 4 , with 1 highest) by a combination of an external magnetic field with the electric field of the trigonal crystal. The magnetic field is tuned in magnitude and direction until levels 2 and 3 are split by the ultrasonic frequency of 12 $\mathrm{GHz}$, and levels 1 and 3 (and 2 and 4 ) by a microwave pump frequency of $24 \mathrm{GHz}$. Before pumping, the population of level 2 is significantly less than that of 3. Pumping reverses this, because it increases the population of 2 while reducing that of 3 . Ultrasonic amplification now takes place by stimulated emission as in a laser.

The levels couple to both ultrasound and microwave radiation, which is not too surprising, as the ultrasound makes the positive nuclei vibrate and this creates oscillating electric fields. In fact, the same system had been developed earlier as a microwave amplifier or maser, and was

\section{Population inversion in the SASER}

THE aim of adiabatic rapid passage (ARP) is to exchange the populations of two levels separated by the energy $h \omega_{0}$ by sweeping the frequency $\omega$ of an oscillating disturbance (the acoustic strain in the present case) through $\omega_{0}$. When $\omega=\omega_{0}$ the amplitudes $p$ and $q$, from which the populations $|p|^{2}$ and $|q|^{2}$ of the two levels are obtained, oscillate with a frequency $\omega_{1}$ which depends on the amplitude of the strain and its coupling to the TLS. More generally

$$
\begin{gathered}
\mathrm{d} p / \mathrm{d} t=-i \omega_{0} p / 2-i \omega_{1} \exp (-i \omega t) q \\
\mathrm{~d} q / \mathrm{d} t=i \omega_{0} q / 2-i \omega_{1} \exp (i \omega t) p
\end{gathered}
$$

By choosing two new variables $p^{\prime}$ and $q^{\prime}$ the equations are transformed into ones of the form $\mathrm{d} p^{\prime} / \mathrm{d} t=i \Omega p^{\prime} / 2$ from which the disturbance has disappeared because it has been incorporated coherently into the TLS. The energy levels of this composite TLS are $\pm h \Omega / 2$ where $\Omega=\left(\left(\omega_{0}-\omega\right)^{2}+\omega_{1}^{2}\right)^{1 / 2}$, and show 'anticrossing' behaviour (see Fig. 1). As $\omega$ changes through $\omega_{0}$ the eigenstates of the composite TLS change between the eigenstates of the original TLS, and the effect is to exchange the latter's populations. The technique requires the change of $\omega$ to be slow enough for the state of the composite TLS to follow, but if the change is
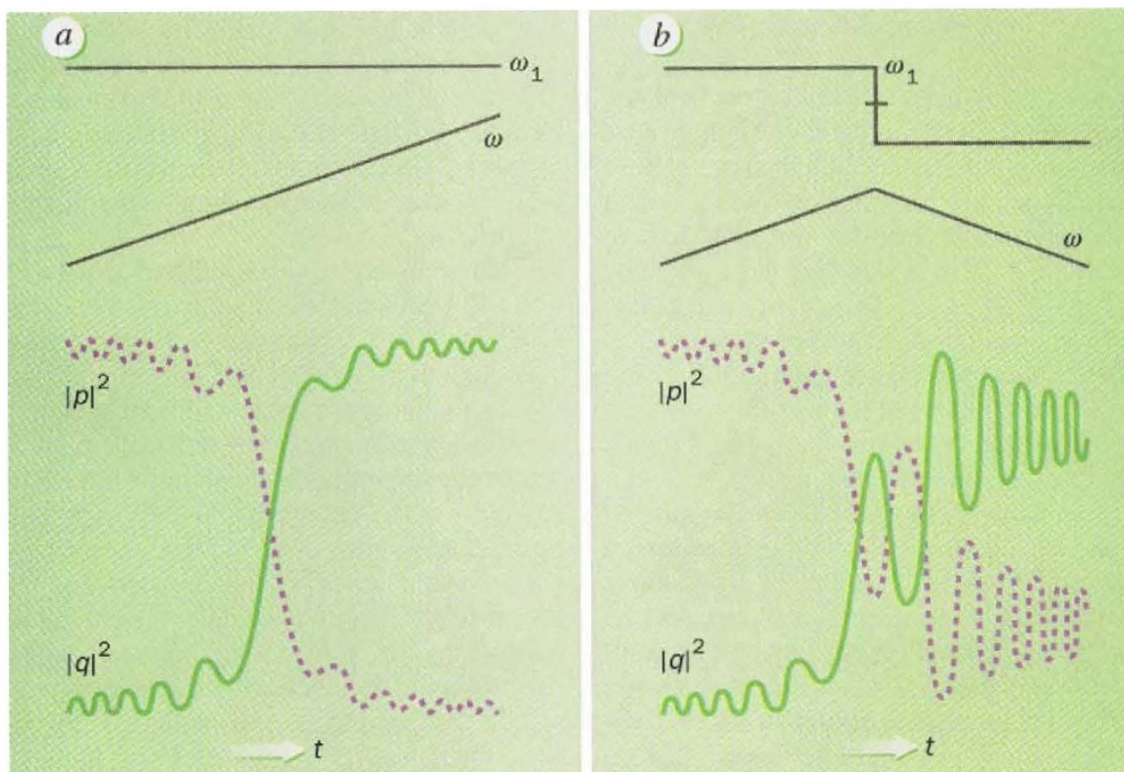

FIG. 2 Simulations of the usual ARP ( $a$ ) and the variant $(b)$ in which the direction of sweep is reversed at $\omega_{0}$ with a phase switch. The sweep speed is as large as can be tolerated while achieving a reasonable degree of inversion.

too slow the population inversion decays because of relaxation processes. A com. promise is necessary. Oscillations of the amplitudes $p$ and $q$ are generated and the
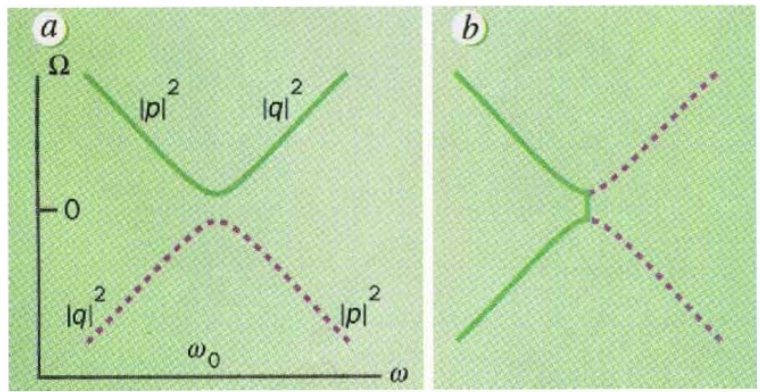

FIG. 1 The levels of the composite TLS exhibit anticrossing which is exploited in ARP. $a$, Usually $\left(\omega-\omega_{0}\right)$ is swept through 0 following the paths shown. $b$, The variant reverses the direction of sweep at 0 . inversion is less than perfect.

Prieur and colleagues' acoustic amplifier uses an unusual variant of ARP in which $\omega$ is swept to $\omega_{0}$ and back again with a sudden switch of phase of $\pi$ where the direction of the sweep is reversed. The usual ARP follows the two paths in Fig. 1a. The route of the variant is shown in Fig. $1 b$. Figure 2 shows simulations of standard ARP and the variant under conditions of compromise between sweep rate and inversion efficiency. The variant only inverts populations for TLS having $\omega_{0}$ close to the extreme value of w. Standard ARP would invert populations over a wider band of TLS frequencies.

L.C. \&S.C. 